\title{
Investigation of the Role of the Acceptor Molecule in Bulk Heterojunction Photovoltaic Cells Using Impedance Spectroscopy
}

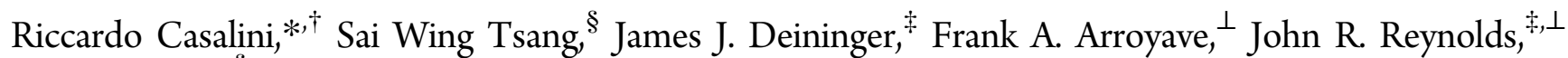
and Franky So ${ }^{\S}$

\author{
${ }^{\dagger}$ Chemistry Division, Code 6120, Naval Research Laboratory, Washington, DC 20375-5342, United States \\ ${ }^{\ddagger}$ School of Chemistry and Biochemistry, School of Materials Science and Engineering, Georgia Institute of Technology, Atlanta, \\ Georgia 30332-0400, United States \\ ${ }^{\S}$ Department of Materials Science and Engineering, and ${ }^{\perp}$ Department of Chemistry, University of Florida, Gainesville, Florida 32611, \\ United States
}

Supporting Information

ABSTRACT: An investigation of the recombination kinetics and the density of states distribution in bulk heterojunction organic photovoltaic cells was undertaken using impedance spectroscopy measurements under varying levels of illuminations. Three organic polymer solar cells were investigated which differed only by the p-type polymer used in the active layers while the device architecture and acceptor molecule (PC70BM) were kept the same. We found that the density of states profiles for the three cells are similar and can be superimposed by a horizontal shift due to a difference between the polymers' HOMO and the PC70BM's LUMO levels. The recombination mechanisms for the three cells showed the same behavior, suggesting an important role of the acceptor in this process; however, large differences in the absolute value of carrier lifetime $\tau_{\text {eff }}$ related to the choice of the polymer are evident, indicating that the recombination kinetics depends on a combination of the properties of both materials and the morphology of their blend.

\section{INTRODUCTION}

Even though the power conversion efficiency of state of the art organic photovoltaics (OPVs) cells is still lower than their inorganic counterparts, there is a growing interest in transforming these cells from laboratory curiosities into commercial products. This interest is fueled by both (i) potential economically advantageous low production costs using fabrication techniques like roll-to-roll processing and inkjet printing and (ii) their potential use in new applications because of their light weight and flexibility. The power conversion efficiency values of single junction OPVs reported in the literature have been fast approaching the $10 \%$ mark, thanks to the many synthetic (i.e., new materials) and processing routes (i.e., morphology optimization, optimization of interfaces, etc.) available in the design of OPVs, which hold promise for further developments.

The most successful device architecture used for OPV cells is based on the bulk heterojunction (BHJ). ${ }^{1,2}$ In $\mathrm{BHJ}$ cells, the active layer consists of a thin film $(\sim 100 \mathrm{~nm}$ thick $)$ of an interpenetrating network of an electron accepting material (generally a fullerene derivative) and an electron donating light absorbing material (generally a semiconducting polymer). $\mathrm{BHJ}$ cells are excitonic solar cells, where excitons (strongly bound hole-electron pairs) are generated by light absorption in the active layer. The strongly bound pairs cannot directly dissociate

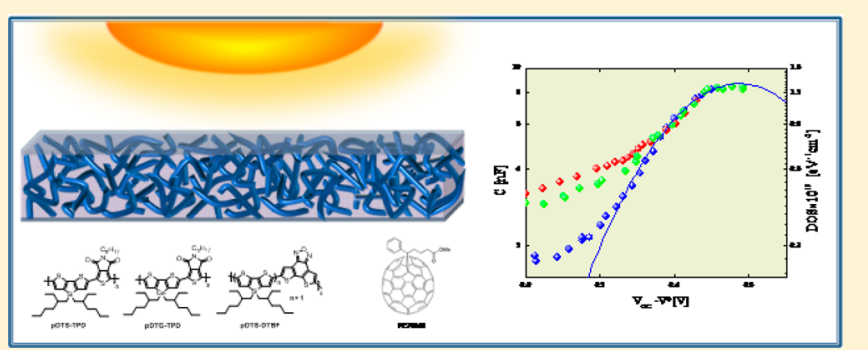

because of the low dielectric constant of the organic materials, and dissociation occurs only at the donor-acceptor interface due to the difference in electron affinities of the two materials (commonly taken to be larger than $0.3 \mathrm{eV}$ ). Since excitons are neutral species, they can move toward the interface only by diffusion, and to obtain an optimal energy conversion, the time necessary to diffuse to the interface has to be smaller than the exciton lifetime. Therefore an ideal morphology is that of a bicontinuous composite in which the interfacial area is maximized and the size of the domains is small $(\sim 20 \mathrm{~nm})$. The efficiency of a BHJ cell is therefore a complicated convolution of different factors; some are intrinsic to the materials and some are related to the architecture of the cell, and a better physical understanding of how these factors influence the final efficiency is fundamental to guiding further improvements.

Herein, we report on an impedance spectroscopic study of a set $\mathrm{BHJ}$ cells with different donor polymers and investigate how the choice of a different donor material affects the density of states and the recombination kinetics. We find that the acceptor

Received: February 8, 2013

Revised: June 4, 2013

Published: June 26, 2013 


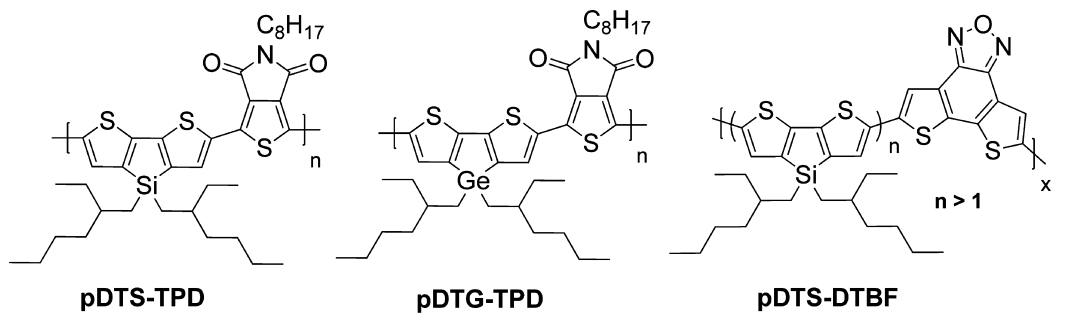

Figure 1. Repeat unit structures of the three semiconducting polymers used in the active layers together with PC70BM.

molecules play an important role in determining the density of states in the band gap and the recombination dynamics.

\section{EXPERIMENTAL SECTION}

The photovoltaic cells used in this study have the following architecture: ITO/ZnO/active layer $/ \mathrm{MoO}_{3} / \mathrm{Ag}$. The three active layers investigated were prepared from mixtures of PC70BM with three different semiconducting polymers (Figure 1): (i) dithienosilole/dithieno $\left[3^{\prime}, 2^{\prime}: 3,4 ; 2^{\prime \prime}, 3^{\prime \prime}: 5,6\right]$ benzo[ $[1,2-c]$ furazan statistical copolymer (pDTS-DTBF) (synthetic and characterization details in the Supporting Information), (ii) dithienogermol/N-octylthienopyrrolodione copolymer (pDTG-TPD), ${ }^{3}$ and (iii) dithienosilole/ $N$-octylthienopyrrolodione copolymer (pDTS-TPD). ${ }^{3}$ The PC70BM was obtained from Nano-C and used as received. Inverted solar cells were processed on prepatterned ITO-coated glass substrates with a sheet resistance of $20 \Omega$ per square. First, a thin layer $(30 \mathrm{~nm})$ of $\mathrm{ZnO}$ solution was spin-coated on top of the ITO glass substrate and annealed at $200{ }^{\circ} \mathrm{C}$ for $40 \mathrm{~min}$ in air. A solution containing a mixture of polymer:PC70BM in chlorobenzene with a concentration of $20 \mathrm{mg} / \mathrm{mL}$ was used and $3 \%$ of volume ratio of diiodoctane (DIO) solvent additive was added to improve the miscibility between the polymer and PC70BM. The blend solution was spin-coated on top of the $\mathrm{ZnO}$ film and followed by annealing at $80{ }^{\circ} \mathrm{C}$ for $30 \mathrm{~min} . \mathrm{MoO}_{3}(5 \mathrm{~nm})$ and silver $(80 \mathrm{~nm})$ were thermally evaporated at a vacuum of $\sim 10^{-7}$ mbar on top of the active layer as an anode. More details are discussed in the Supporting Information of our previous publication. $^{3}$ The area of the cells was $4.6 \mathrm{~mm}^{2}$, and the thickness of the active layer was $\sim 100 \mathrm{~nm}$. The cells were prepared at the University of Florida and encapsulated using epoxy. The cell performance was fully characterized using an AM 1.5 G solar simulator with irradiation intensity of $100 \mathrm{~mW}$ $\mathrm{cm}^{-2}$ before sending to NRL.

The impedance of the devices was measured using a Novocontrol Alpha impedance analyzer with a signal amplitude $V_{\text {ac }}=15 \mathrm{mV}$ under a dc bias. The low $V_{\mathrm{ac}}$ value is necessary because of the nonlinearity of the OPV cells' behavior (diodelike behavior). Impedance measurements were performed under different conditions of illumination, controlled by varying the input voltage to the light source. The open circuit voltage, $V_{o c}$ was measured before connecting the device to the impedance analyzer, and a bias voltage equal to $V_{\text {oc }}$ was applied during the impedance measurement so that the impedance was measured in open circuit condition. ${ }^{4}$ During the measurements the samples were kept under a nitrogen atmosphere.

\section{RESULTS AND DISCUSSION}

Figure 1 shows the molecular structures of the polymers investigated for this study. Solar cells were fabricated using the three polymers, and the corresponding $I-V$ curves measured under 1 sun illumination are reported in Figure 2 . The photovoltaic parameters extracted from the $I-V$ curves,

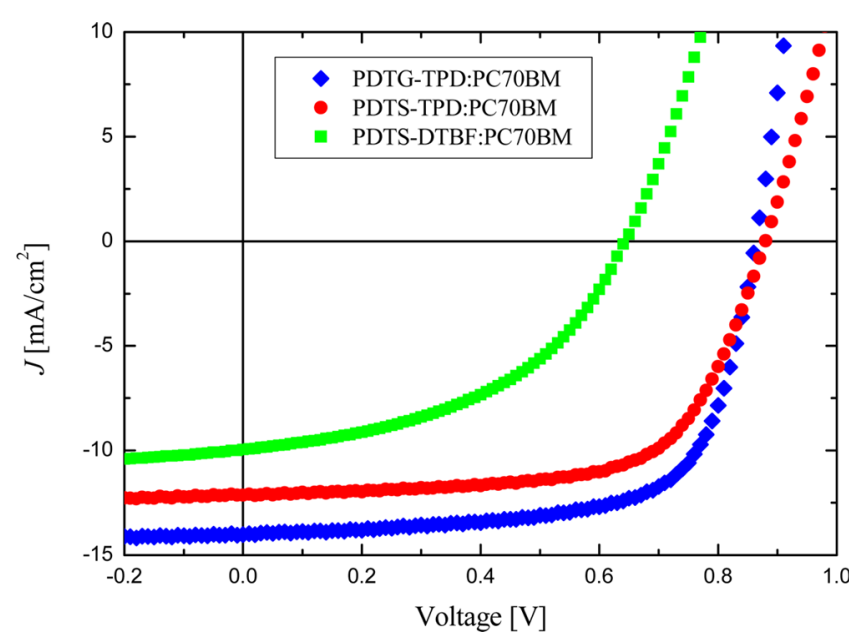

Figure 2. Current density as a function of applied voltage under 1 sun illumination for the three cells investigated, in which the only difference was the polymer used. The cells' parameters extracted from these results are reported in Table 1 . The device data of pDTS-TPD and pDTG-TPD have been previously reported in ref 3 .

together with the known HOMO and LUMO levels, are reported in Table 1. Figure 3 shows representative impedance spectra for the cell with pDTS-TPD measured under different conditions of illumination with a bias $V_{\text {bias }}=V_{\text {oc }}$. The spectra show a decrease of several orders of magnitude of the impedance with increasing illumination. The frequency dependence of the real and imaginary parts of the impedance is described quite well by an equivalent circuit constituted by a parallel $R C$ network in series with a smaller resistance $r_{\mathrm{s}}$

$$
Z^{*}(f)=\frac{R}{1+(i 2 \pi f R C)}+r_{\mathrm{s}}
$$

where $r_{s}$ is mainly related to the electrodes' resistance, while the resistance $R$ and the capacitance $C$ are related to the recombination kinetics and the density of states according to the model of Garcia-Belmonte and co-workers. ${ }^{4-7}$ The solid lines in Figure 3 are the best fit of the spectra using this equivalent circuit model (eq 1). Reported in the insets to Figure 3 are the Nyquist plots of the same data along with their best fits. A semicircle in a Nyquist plot is indicative of an equivalent circuit with a single parallel $R C$ network; thus, no additional processes are present in the spectra.

The description of the spectra using eq 1 has some limits especially at high frequency where the frequency dependence of $C$ and $R$ cannot be neglected. However we will not discuss this dependence herein, but we focus mainly on the low frequency 
Table 1. Solar Cell Performance Parameters ${ }^{a}$

\begin{tabular}{lccccc} 
polymer & acceptor & $J_{\text {SC }}\left(\mathrm{mA} \mathrm{cm}^{-2}\right)$ & $V_{\text {OC }}(\mathrm{V})$ & FF $(\%)$ & PCE \\
pDTS-DTBF $($ HOMO $=$ NA) & PC70BM (LUMO $=-4.3 \mathrm{eV})$ & 9.8 & 0.64 & 47 \\
pDTG-TPD (HOMO $=-5.6 \mathrm{eV})$ & PC70BM (LUMO $=-4.3 \mathrm{eV})$ & 12.6 & 0.85 & 68 \\
pDTS-TPD $($ HOMO $=-5.6 \mathrm{eV})$ & PC70BM (LUMO $=-4.3 \mathrm{eV})$ & 11.5 & 0.89 & $6.3 \%$ \\
\hline
\end{tabular}

${ }^{a}$ The pDTG-TPD and pDTS-PD data have been previously reported.3

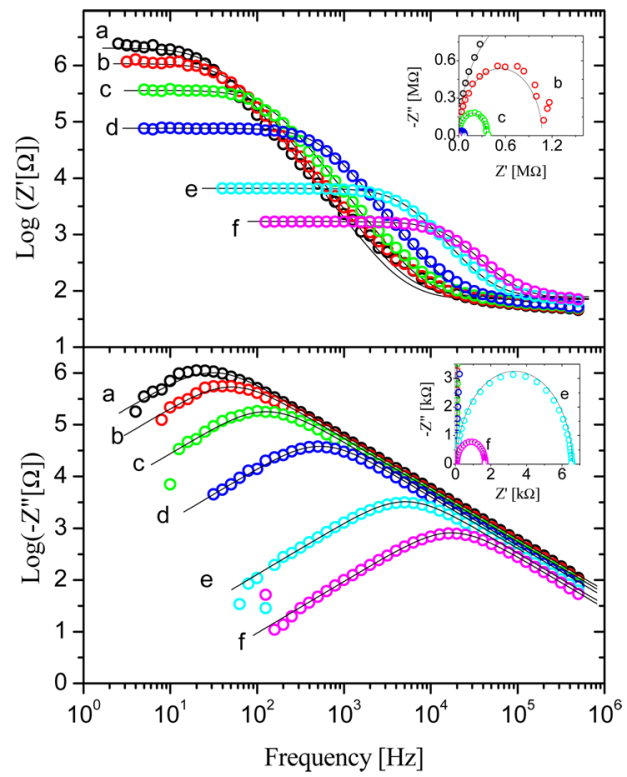

Figure 3. Real and imaginary part of the impedance of the PDTSTPD-PC70BM cell under different level of illumination with $V_{\text {oc }}=$ 0.220 (a), 0.350 (b), 0.485 (c), 0.569 (d), 0.663 (e), and 0.726 (f) V (from left to right). The solid lines are the best fit using eq 1 . The insets are Nyquist plots of the same data and best fits in the main figure (with the same colors). A single semicircumference in a Nyquist plots indicates that the equivalent circuit is an $R-C$ parallel.

response and its description using eq 1 . This is equivalent to determining the value of $R$ and $C$ at low frequency $(\sim 10 \mathrm{~Hz})$.

The capacitance $C$ is related to two main contributions in parallel to each other, and both are bias dependent. The first contribution is related to the depletion layer capacitance at the metal interface, which increases with the bias up to its maximum value at the flat band condition: $V_{\text {bias }}=V_{\mathrm{fb}}=\left(\phi_{\mathrm{c}}-\right.$ $\left.\phi_{\mathrm{s}}\right) / e$, where $\phi_{\mathrm{c}}$ is the metal work function and $\phi_{\mathrm{s}}$ is the active layer Fermi level. The second contribution to the capacitance is the chemical capacitance $C_{\mu}$ which is related to the change in the charge density in the active layer, $n$, due to the displacement of the electrochemical potential ${ }^{4}$

$$
C_{\mu}=A t e^{2} \frac{\partial n}{\partial E_{\mathrm{Fn}}}
$$

where $e$ is the electron charge, $E_{\mathrm{Fn}}$ is the Fermi level of the acceptor material, $A$ is the area of the cell, and $t$ is the thickness of the active layer. The variation of the capacitance as a function of $V_{\text {oc }}$ (Figure 4) shows two different zones: at small $V_{\text {oc }}$ where $C$ is dominated by the depletion capacitance and at large $V_{\text {oc }}$ where the chemical capacitance becomes dominant. ${ }^{4}$

On the basis of thermodynamic principles, the open circuit voltage $V_{\mathrm{oc}}$ is related to the difference between $E_{\mathrm{Fn}}$ and the Fermi level of the donor polymer, $E_{\mathrm{Fp}}:{ }^{8}$

$$
e V_{\mathrm{oc}}=E_{\mathrm{Fp}}-E_{\mathrm{Fn}}
$$

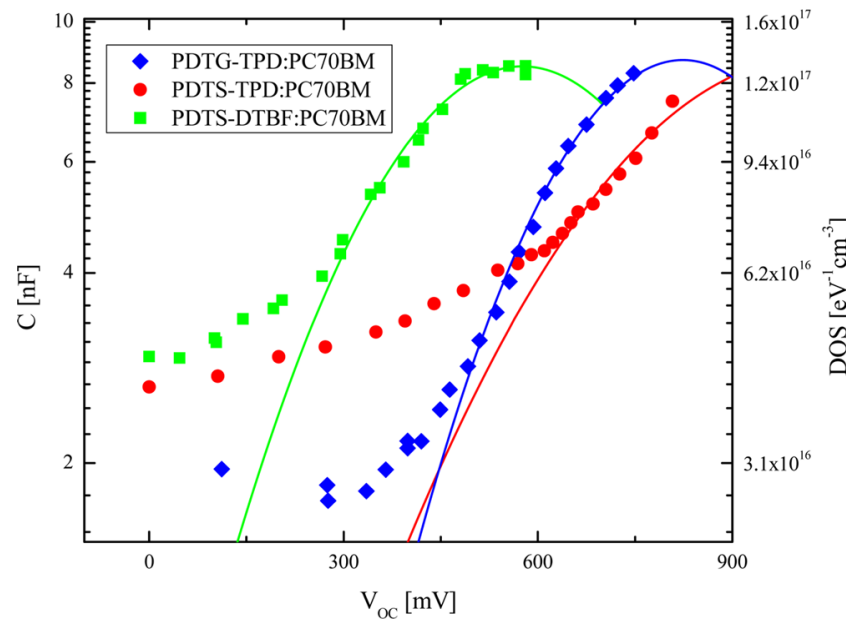

Figure 4. Capacitance as a function of open circuit voltage measured under illumination for the three devices. The solid lines are the best fit to a Gaussian distribution. On the right axis the DOS of states is calculated from the capacitance as discussed in the text.

Therefore, the theoretical upper limit of $V_{\mathrm{oc}}$ is equal to the difference between the LUMO of the fullerene and the HOMO of the polymer. This is in agreement with the observation that for BHJ solar cells with various combinations of donor/ acceptor the open circuit voltage under 1 sun of illumination is found to be close to the difference between the HOMO of the donor and the LUMO of the acceptor. ${ }^{9}$ Deviation from the behavior predicted from eq 3 , reporting a smaller $V_{\text {oc }}$ than expected, has been interpreted as due to the recombination of dissociated charges. ${ }^{10}$

Because of the broadening of the energy level due to the intrinsic disorder in these systems, for low level of illumination, the available states are those lying at the lower energy level of the fullerene's LUMO; then with increasing $V_{\mathrm{oc}}$ (increasing illumination) the $E_{\mathrm{Fn}}$ increases, and band gap states with increasing energy are progressively occupied by charges and these charges contribute to $C_{\mu}$. The $E_{\mathrm{Fp}}$ can be assumed constant because the initial charge density prior to illumination $p_{0}$ is much higher than its variation under illumination such that $p \sim p_{0}$. Using the zero temperature approximation of the Fermi function at occupancy higher than $1 \% C_{\mu}$ is proportional to the density of states, $g\left(E_{\mathrm{Fn}}\right)^{4,11}$

$$
C_{\mu}=\operatorname{Ate}^{2} g\left(E_{\mathrm{Fn}}\right)
$$

Assuming that the density of the states exhibits a Gaussian distribution, we then expect

$$
C_{\mu}=A t e^{2} \frac{N}{\sqrt{2 \pi} \sigma} \exp \left[-\frac{\left(E_{\mathrm{Fn}}-E_{0}\right)^{2}}{2 \sigma^{2}}\right]
$$

where $N$ accounts for the total DOS with a mean energy $E_{0}$ and width $\sigma$. 
When analyzing $C_{\mu}$ with a Gaussian distribution, we expect that the maximum of $C_{\mu}$ (i.e., $E_{0}$ ) will be close to the difference between the LUMO of PCBM and the HOMO of the polymers, while $\sigma$ and $N$ give a measure of the disorder and the value of the DOS of the PCBM lying in the band gap. In our case for all of the investigated devices, since we used the same fullerene derivative while changing the polymer, we would expect mainly a shift in $E_{0}$ with no large differences in the shape of the distribution assuming that no additional disorder is introduced by the different states formed at the interface between the polymer and PCBM.

The solid lines in Figure 4 are the best fit of the capacitance for large bias using eq 5 , obtained for the parameters reported in Table 2. These measurements are in good agreement with

Table 2. Best Fit Parameters of $C_{\mu}$ Data in Figure 3 with a Gaussian Distribution

$\begin{array}{lccc} & E_{0}(\mathrm{eV}) & \sigma(\mathrm{eV}) & N\left(\mathrm{~cm}^{-3}\right) \\ \text { pDTS-DTBF:PC70BM } & 0.6 & 0.24 & 7.4 \times 10^{16} \\ \text { pDTS-TPD:PC70BM } & 1.0 & 0.33 & 1.1 \times 10^{17} \\ \text { pDTG-TPD:PC70BM } & 0.8 & 0.22 & 7.8 \times 10^{16}\end{array}$

the expectation that the difference in behavior should be mainly due to a shift in $E_{0}$. For the case of pDTS-TPD the values of $\sigma$ and $N$ are quite different from the other two polymers; however, observing the data it can be seen that the range in which the best fit can be made is also more limited because of a larger contribution of the depletion capacitance. To compare the different sets of data without any fitting, Figure 5 shows the

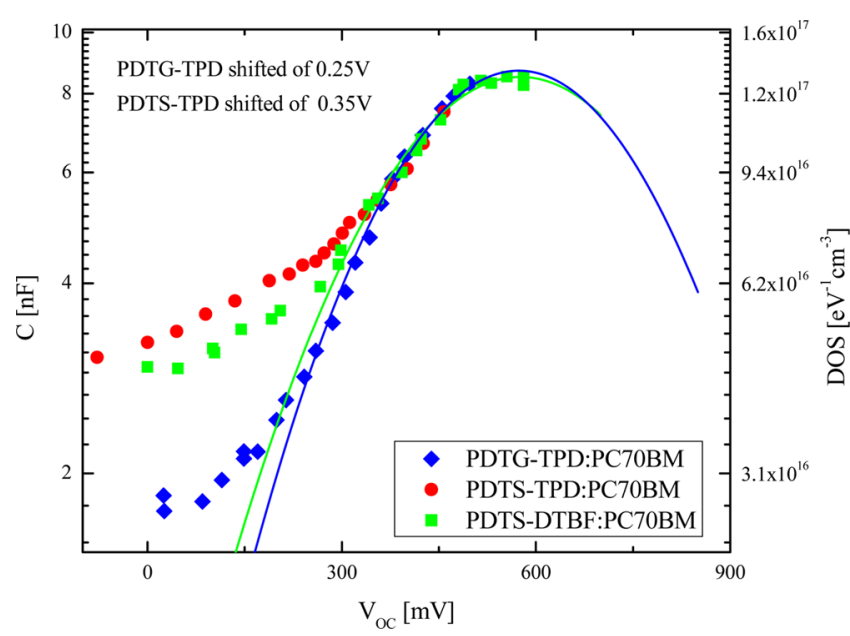

Figure 5. Capacitance as a function of open circuit voltage measured under illumination for the three devices as reported in Figure 4 with the $x$-axis shifted by the value reported in the figure for the PDTGTPD and PDTS-TPD.

same data reported in Figure 4, but shifting the data of the devices of pDTS-TPD and pDTG-TPD along the $x$-axis by, respectively, 0.35 and $0.25 \mathrm{~V}$, to superimpose with the data of pDTS-DTBF without any vertical shift. Figure 5 clearly shows that the $C_{\mu}$ data at high $V_{\mathrm{oc}}$ have the same behavior and value for all three materials as expected if $C_{\mu}$ is determined by the DOS of the PC70BM that is common to all three devices. These results are in some ways complementary with the results found in a series of devices made using the same polymer (P3HT) with different acceptors. ${ }^{6}$ In that case, different from ours, it was found that the variation of $C_{\mu}$ vs $V_{\mathrm{oc}}$ was distinct between the different cells. ${ }^{6}$

Using cyclic voltammetry, it has been found ${ }^{3}$ that the pDTSTPD and pDTG-TPD HOMOs are minimally different: $\mathrm{HOMO}(\mathrm{pDTS}-\mathrm{TPD})-\mathrm{HOMO}(\mathrm{pDTG}-\mathrm{TPD})=0.05 \mathrm{eV}$, therefore quite close to the difference of the shift necessary to superimpose the DOS curves in Figure 5, and a deviation certainly smaller than the deviations observed from eq $3 .^{9}$ While the HOMO of the pDTS-DTBF is not available, from a point of view of the structure since the HOMO level should be mainly determined by the DTS group, it is expected that the HOMO of pDTS-DTBF should be close to that of pDTS-TPD. But the $V_{\text {oc }}$ of the cells with pDTS-DTBF under 1 sun illumination is $0.25 \mathrm{~V}$ lower than that of pDTS-TPD. According to eq 3 the difference of $V_{\mathrm{oc}}$ and the difference $\mathrm{HOMO}$ (pDTSTPD) - HOMO(pDTS-BTBF) should be $\sim 0.25 \mathrm{eV}$. This value is indeed quite close to the difference $0.35 \mathrm{eV}$, determined from the shift necessary to superimpose the two DOS curves in Figure 5.

Impedance measurements allow a simultaneous investigation of both the DOS and the recombination dynamics. ${ }^{4}$ While as discussed above the value of the DOS can be extracted from the capacitance, the information about the recombination dynamics can be obtained from the resistance $R$. In the conditions for which the capacitance is dominated by the chemical capacitance (i.e., at large $V_{\mathrm{oc}}$ ), the resistance $R$ is dominated by the recombination resistance $R_{\text {rec }}{ }^{4}$

$$
R_{\mathrm{rec}}=\frac{1}{e^{2}}\left(\frac{\partial U}{\partial E_{\mathrm{Fn}}}\right)^{-1}
$$

where $U$ is the recombination rate. Figure 6 shows the behavior of the resistance as a function of the open circuit voltage.

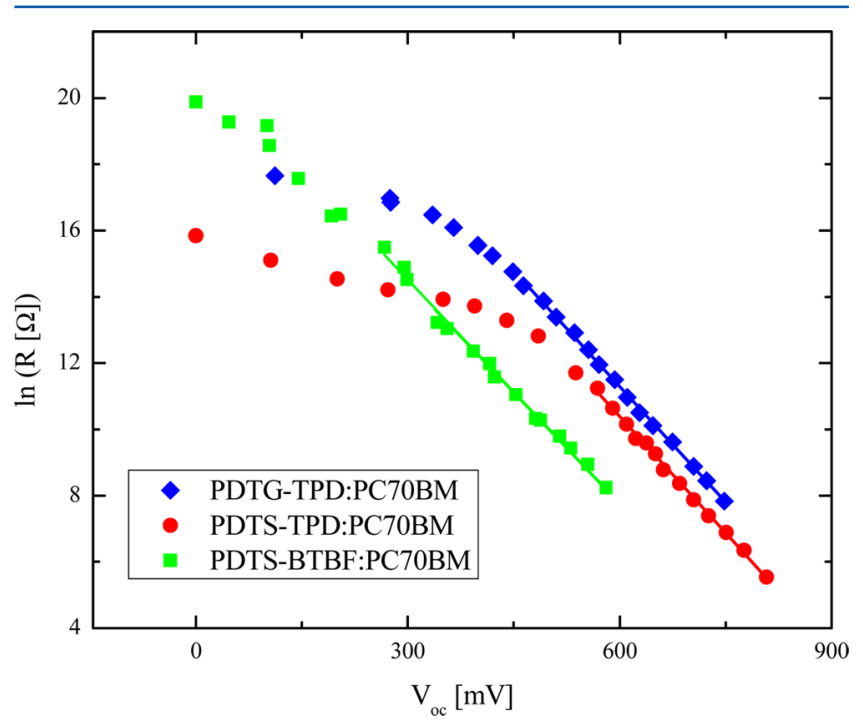

Figure 6. Recombination resistance as a function of open circuit voltage under different conditions of illumination. The solid lines are the fits to an exponential function with $\beta=0.58,0.58$, and 0.56 for pDTG-TPD, pDTS-TPD, and pDTS-DTBF, respectively.

Similar to the behavior of the capacitance (Figure 4) the behavior of $R$ shows two ranges, one dominated by the interface at low $V_{\text {oc }}$ and the other at higher voltages where the resistance is related to the recombination resistance, $R_{\text {rec }}$. At high $V_{\text {oc }}$ the $R_{\text {rec }}$ value varies nearly exponentially with $V_{\text {oc }}$. The 
best fit of the $R$ data at large $V_{\text {oc }}$ (solid lines in Figure 6) with the function $R_{\mathrm{rec}}=R_{0} \exp \left(-\beta V_{\mathrm{oc}} / k T\right)$ was obtained for $\beta=0.58$ \pm 0.1 for all three devices; this value is close to that found by others for similar devices. ${ }^{7}$ Between the three devices the main difference is a shift in $V_{\text {oc }}$ like for the case of the capacitance; however, the shifts are not the same as those necessary to superimpose the capacitance curves. The shifts necessary to superimpose the data of pDTG-TPD and pDTS-TPD to pDTS-DTBF are 0.16 and $0.12 \mathrm{~V}$, respectively, therefore much smaller than in the case of $C_{\mu}$. This difference, however, is not surprising since we would expect that additional factors such as the charge mobility in the polymer will affect the recombination dynamics leading to a shift in $V_{\text {oc }}$. The importance of the polymer properties in determining the cell recombination rate is also shown in a recent study using impedance spectroscopy for three cells having active layers prepared using the same polymer (P3HT) mixed with three different acceptors (PC60BM, PC70BM, and ICBA). ${ }^{12}$ In this case the shifts in $V_{\mathrm{oc}}$ for $C_{\mu}$ and $R_{\mathrm{rec}}$ are the same and correlate with the energetic difference between HOMO of the donor and the LUMO of the acceptor (eq 3 ).

From the product of the recombination resistance and the capacitance, we calculated the charge carrier lifetime: ${ }^{4}$

$$
\tau_{\text {eff }}=\left(\frac{\partial U}{\partial n}\right)^{-1}=R_{\text {rec }} C_{\mu}
$$

Comparing the results for the three devices (Figure 7), we observe that the recombination lifetime decreases many orders

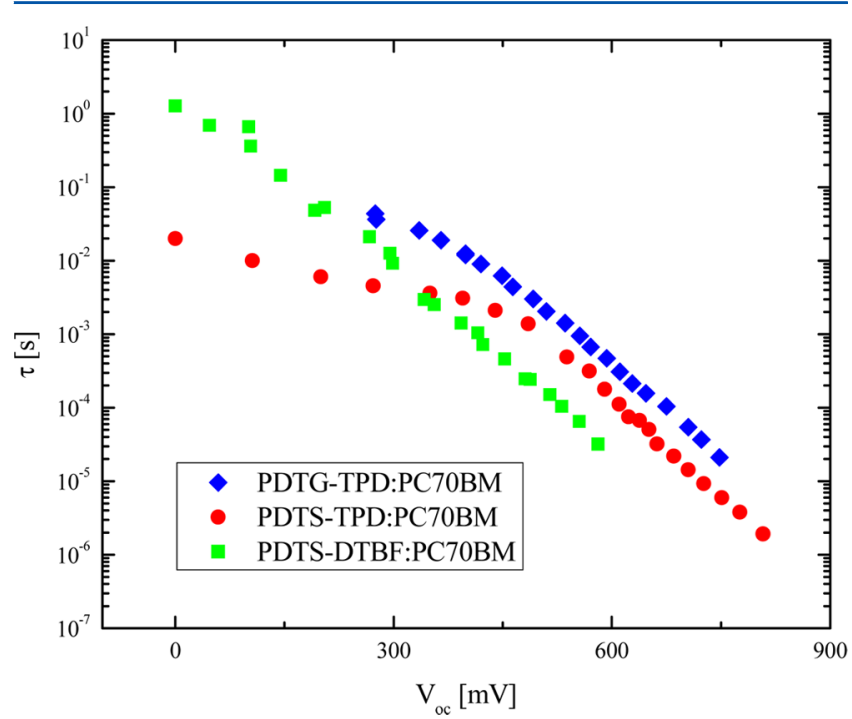

Figure 7. Carrier lifetime as a function of open circuit voltage under different conditions of illumination.

of magnitude with increasing illumination. Evidently $\tau_{\text {eff }}$ behavior is mainly dominated by the dependence of $R_{\text {rec }}$ on illumination. At the same $V_{\text {oc }}$ the carrier lifetime is longer for the PDTG-TPD cell by more than 1 order of magnitude than the less efficient cell with PDTS-BTBF, but their slopes at high $V_{\text {oc }}$ are very close. For all three devices the behavior at high bias is well described by the same exponential behavior $\tau_{\text {eff }}=\tau_{0} \exp$ $\left(-V_{\text {oc }} / b k T\right)$ with $b=2.0 \pm 0.1$ for all three devices. Substituting eq 3 , we find that for all three cases $\tau_{\text {eff }} \sim \tau_{0} \exp$ $\left[-\left(E_{\mathrm{Fn}}-E_{\mathrm{Fp}}\right) / 2 k T\right]$.

To investigate the recombination mechanism, it is important to determine the relationship between the recombination dynamics and the charge carrier density. We calculated the charge carrier density by integrating the capacitance $C_{\mu}$ according to ${ }^{4,13}$

$$
n=\frac{1}{e L} \int_{0}^{V_{o c}} C_{\mu}(V) \mathrm{d} V
$$

Considering that the chemical capacitance dominates the measured capacitance only at high bias, we calculated $n$ not as the integral of $C$ but as the integral of the Gaussian fits. However, we observed that this is a minor correction since a calculation using the integral of the experimental values of the measured capacitance $C$ over the entire $V_{o c}$ range gives essentially the same behavior.

For the case of bimolecular recombination the carrier lifetime and the charge carrier density are related by the relationship ${ }^{4,12}$

$$
\tau_{\text {eff }}=\frac{1}{2 \gamma n}
$$

where $\gamma$ is the recombination coefficient. Thus, for the case in which $\gamma$ is independent of the charge density (like for the Langevin recombination in a homogeneous system) a slope -1 is expected in a plot of $\log (\tau)$ vs $\log (n)$.

The behavior of the charge lifetime versus the charge density suggests the presence of two regimes: (i) The first at low $n$, where the slope is close to -1 (solid lines in Figure 8),

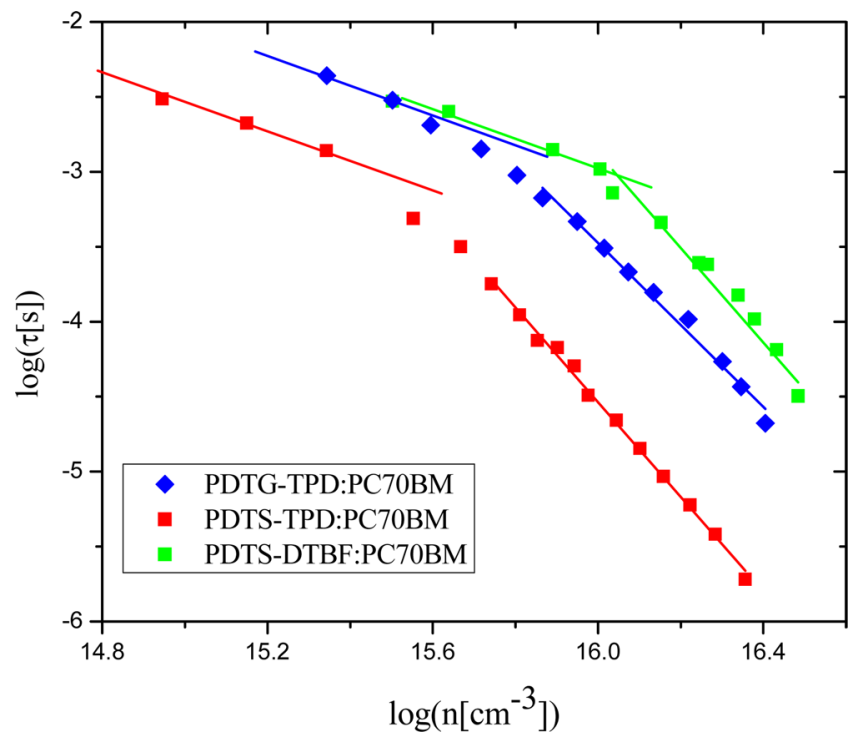

Figure 8. Carrier lifetime as a function of charge carrier density. The solid lines at low $n$ are guides to the eye showing the slope -1 , the solid lines at high $n$ are the best fit to the data this range, with slopes between $-2.7 \pm 0.2$ and $-3.1 \pm 0.2$.

corresponding to a constant recombination coefficient $\gamma$. However, as discussed before, the behavior of $C$ and $R$ (and therefore the calculated $\left(\tau_{\text {eff }}\right)$ in this regime) is dominated by the active layer/metal interface rather than the active layer properties, so the significance of these data is somewhat limited. (ii) At higher light intensity the exponents, $\lambda$, of the power laws describing the data of $\log (\tau)$ vs $\log (n)$ are all close to 3 for all three devices $(2.7 \pm 0.2,3.1 \pm 0.1,3.1 \pm 0.2$ for $\mathrm{pDTG}-\mathrm{TPD}$, pDTS-TPD, and pDTS-DTBF, respectively) indicating that the recombination coefficient is strongly dependent on $n(\gamma \sim$ $\left.n^{\lambda-1}\right)$. Since the slope $\lambda$ is very close for all cases, this result suggests that the recombination kinetics mechanism depends 
more on the fullerene than on the polymer, while the choice of the polymer still influences the absolute value of $\tau_{\text {red }}$ at least for identical architectures like the one used in this case. Power exponents larger than 1 have been reported for several other OPVs with other materials. ${ }^{13,14}$ IS measurements for $\mathrm{BHJ}$ with P3HT/PC70BM found $\lambda \sim 2 ;^{13}$ thus, the exponent cannot be related to just the acceptor and that other details in the morphology and cell architecture have to play a role. A power law behavior with $\lambda \sim 3$ has been reported also from transient photovoltage measurements in $\mathrm{BHJ}$ with poly[2,6-(4,4-bis-(2ethylhexyl)-4H-cyclopenta $\left[2,1-b ; 3,4-b^{\prime}\right]$ dithiophene)-alt-4,7(2,1,3-benzothiadiazole)(PCPDTBT)/PC70BM and poly$[(4,40$-bis (2-ethylhexyl)dithieno[3,2-b:20,30-d] silole)-2,6-diylalt-(4,7-bis(2-thienyl)-2,1,3-benzothiadiazole)-5,50-diyl](SiPCPDTBT)/PC70BM blends for the active layers. ${ }^{10}$ The same authors also found a much lower exponent, $\lambda \sim 1.3$, for $\mathrm{BHJ}$ cells with P3HT/PC60BM and poly(3-hexylselenophene)/ PC60BM. $^{10}$ The smaller value of $\lambda$ was attributed to the larger phase segregation in the cells with PC60BM. BHJ cells with smaller domains will have a larger interfacial area and therefore an increased recombination probability through defects at the interface between donor and acceptor. Thus, the exponent $\lambda \sim$ $2,{ }^{12}$ for a cell with PC70BM, could be indicative of a tendency of PC70BM to form a more segregated phase morphology in blends with $\mathrm{P} 3 \mathrm{HT}$ than with the polymers used here. The bimolecular recombination in $\mathrm{BHJ}$ is significantly smaller than what is expected from the Langevin model, ${ }^{15,16}$ and also this difference has been attributed in part to the morphology of the phase-separated blend.

For the devices studied herein, the efficiency measured under 1 sun of illumination was $7.3 \%, 6.6 \%$, and $3.2 \%$ for pDTGTPD, pDTS-TPD, and pDTS-DTBF, respectively. Generally, higher efficiency is expected for devices with larger difference between the LUMO of the acceptor and the HOMO of the polymer, since this results in a larger $V_{o c}$ and in fact the cell having the smaller $V_{\text {oc }}$ is also less efficient. However, for the cells with pDTG-TPD and pDTS-TPD even if the two polymers have similar HOMO, the pDTG-TPD is significantly more efficient, even if its $V_{\text {oc }}$ is smaller than the pDTS-TPD. ${ }^{3}$ From the IS measurements we find that the $\tau_{\text {eff }}$ of the pDTGTPD device is about an order of magnitude larger than for the pDTS-TPD device (Figures 7 and 8), indicating that the larger efficiency in the pDTG-TPD can be attributed, at least in part, to a slower free charge recombination.

\section{CONCLUSIONS}

Impedance spectroscopy measurements under illumination were performed on BHJ OPV cells with three different polymers and the same acceptor PC70BM. From the IS, information on the DOS and the recombination kinetics was extracted using the framework proposed by Belmonte and coworkers. ${ }^{4}$ We found that the DOS and recombination kinetics in the investigated devices are similar notwithstanding the difference in energetics of the polymers used. In particular the behaviors and values of $C_{\mu}$ for the three different devices at high $V_{\mathrm{oc}}$ are superimposed only by a translation of the $V_{\mathrm{oc}}$-axis. This difference in behavior for the different devices can be attributed to the different HOMO level of the polymers, which causes (eq 3) a shift in $V_{\text {oc }}$. The values of the shifts required to superimpose the DOS curves (Figure 5) are well correlated with the difference in $V_{\text {oc }}$ under 1 sun measured for these devices. $^{3}$
The superposition of the capacitance measurements after shifting the $x$-axis indicates that the DOS distributions are remarkably similar between the different devices. Since the main difference between the devices is the polymer, this result confirms that the relevant DOS distribution depends primarily on the acceptor molecule used in the active layers of the three cells (in this case PC70BM).

The acceptor molecule also appears to play an important role in the determination of the recombination mechanism, since all three devices show an almost identical dependence of $\tau_{\text {eff }}$ on $n$ independently of the polymer used. However, since superpositioning of the $\tau_{\text {eff }}$ vs $V_{\text {oc }}$ curves cannot be obtained with the same shift of the $C_{\mu}$ curves, the choice of the polymers has a relevant influence on the charge carrier lifetime, $\tau_{\text {eff }}$ In fact for the case of cells with pDTG-TPD, pDTS-TPD even if their energetic levels are very similar, the cell with pDTG-TPD has a significantly larger $\tau_{\text {eff }}$ and also higher energy efficiency. Comparison with other data in the literature also indicates that the role of the morphology in the recombination needs to be considered.

From the results herein, we find that the choice of the acceptor molecule (PC70BM in this case) is important in determining not only the $V_{\mathrm{oc}}$ but also the recombination dynamics and the charge carrier density, all crucial factors that need to be optimized to improve the OPVs performance. IS evidently has the advantage of allowing the measurements of all three factors simultaneously and therefore is an optimal tool for the characterization and understanding of the OPVs behavior. Future investigations on a larger number of polymers and acceptors are necessary to clarify the general validity of these results.

\section{ASSOCIATED CONTENT}

\section{Supporting Information}

Additional information on the synthetic procedude and characterization of the Poly(DTS-DTBF). This material is available free of charge via the Internet at http://pubs.acs.org.

\section{AUTHOR INFORMATION}

\section{Corresponding Author}

*E-mail: riccardo.casalini@nrl.navy.mil.

\section{Notes}

The authors declare no competing financial interest.

\section{ACKNOWLEDGMENTS}

R.C. acknowledges the support of the Office of Naval Research (grant N0001411WX21295). J.R.R. and F.S. acknowledge the support of the Office of Naval Research (contract N000141110245) for the fabrication and characterization of the polymer solar cells used in this work.

\section{REFERENCES}

(1) Yu, G.; Gao, J.; Hummelen, J. C.; Wudl, F.; Heeger, A. J. Polymer Photovoltaic Cells-Enhanced Efficiencies via a Network of Internal Donor-Acceptor Heterojunctions. Science 1995, 270, 1789-1791.

(2) Halls, J. J. M.; Walsh, C. A.; Greenham, N. C.; Marseglia, E. A.; Friend, R. H.; Moratti, S. C.; Holmes, A. B. Efficient Photodiodes from Interpenetrating Polymer Networks. Nature 1995, 376, 498-500.

(3) Amb, C. M.; Chen, S.; Graham, K. R.; Subbiah, J.; Small, C. E.; So, F.; Reynolds, J. R. Dithienogermole as a Fused Electron Donor in Bulk Heterojunction Solar Cells. J. Am. Chem. Soc. 2011, 133, 1006210065 . 
(4) Garcia-Belmonte, G.; Boix, P. P.; Bisquert, J.; Sessolo, M.; Bolik, H. J. Simultaneous Determination of Carrier Lifetime and Electron Density-of-States in P3HT:PCBM Organic Solar Cells under Illumination by Impedance Spectroscopy. Sol. Energy Mater. Sol. Cells 2010, 94, 366-375.

(5) Bisquert, J.; Garcia-Belmonte, G. On Voltage, Photovoltage, and Photocurrent in Bulk Heterojunction Organic Solar Cells. J. Phys. Chem. Lett. 2011, 2, 1950-1964.

(6) Garcia-Belmonte, G.; Boix, P. P.; Bisquert, J.; Lenes, M.; Bolink, H. J.; La Rosa, A.; Filippone, S.; Martin, N. Influence of the Intermediate Density-of-States Occupancy on Open-Circuit Voltage of Bulk Heterojunction Solar Cells with Different Fullerene Acceptors. J. Phys. Chem. Lett. 2010, 1, 2566.

(7) Boix, P. P.; Ajuria, J.; Etxebarria, I.; Pacios, R.; Garcia-Belmonte, G.; Bisquert, J. Role of ZnO Electron-Selective Layers in Regular and Inverted Bulk Heterojunction Solar Cells. J. Phys. Chem. Lett. 2011, 2, 407-411.

(8) Bisquert, J.; Cahen, D.; Ruhle, S.; Hodes, G.; Zaban, A. Physical Chemical Principles of Photovoltaic Conversion with Nanoparticulate, Mesoporous Dye-Sensitized Solar Cells. J. Phys. Chem. B 2004, 108, 8106.

(9) Scharber, M. K.; Mühlbacher, D.; Koppe, M.; Denk, P.; Waldauf, C.; Heeger, A. J.; Brabec, C. J. Design Rules for Donors in BulkHeterojunction Solar Cells-Towards 10\% Energy-Conversion Efficiency. Adv. Mater. 2006, 18, 789-794.

(10) Maurano, A.; Hamilton, R.; Shuttle, C. G.; Ballantyne, A. M.; Nelson, J.; O’Regan, B.; Zhang, W.; McCulloch, I.; Azimi, H.; Morana, M.; Brabec, C. J.; Durrant, J. R. Recombination Dynamics as a Key Determinant of Open Circuit Voltage in Organic Bulk Heterojunction Solar Cells: A Comparison of Four Different Donor Polymers. Adv. Mater. 2010, 22, 4987-4992.

(11) Pomerantz, Z.; Zaban, A.; Ghosh, S.; Lellche, J.-P.; GarciaBelmonte, G.; Bisquert, J. Capacitance, Spectroelectrochemistry and Conductivity of Polarons and Bipolarons in a Polydicarbazole Based Conducting Polymer. J. Electroanal. Chem. 2008, 614, 49-60.

(12) Guerrero, A.; Marchesi, L. F.; Boix, P. P.; Bisquert, J.; GarciaBelmonte, G. Recombination in Organic Bulk Heterojunction Solar Cells: Small Dependence of Interfacial Charge Transfer Kinetics on Fullerene Affinity. J. Phys. Chem. Lett. 2012, 3, 1386.

(13) Zhang, Y.; Dang, X.; Kim, C.; Nguyen, T. Effect of Charge Recombination on the Fill Factor of Small Molecule Bulk Heterojunction Solar Cells. Adv. Energy Mater. 2011, 1, 610-617.

(14) Shuttle, C. G.; O’Regan, B.; Ballantyne, A. M.; Nelson, J.; Bradley, D. D. C.; de Mello, J.; Durrant, J. R. Experimental Determination of the Rate Law for Charge Carrier Decay in a Polythiophene: Fullerene Solar Cell. Appl. Phys. Lett. 2008, 92, 093311.

(15) Clarke, T. M.; Peet, J.; Denk, P.; Dennler, G.; Lungenschmied, C.; Mozer, A. J. Non-Langevin Bimolecular Recombination in s SiloleBased Polymer:PCBM Solar Cell Measured By Time-Resolved Charge Extraction and Resistance-Dependent Time-Of-Flight Techniques. Energy Environ. Sci. 2012, 5, 5241-5245.

(16) Clarke, T. M.; Rodovsky, D. B.; Herzing, A. A.; Peet, J.; Dennler, G.; De Longchamp, D.; Lungenschmied, C.; Mozer, A. J. Significantly Reduced Bimolecular Recombination in a Novel Silole-Based Polymer: Fullerene Blend. Adv. Energy Mat. 2011, 1, 1062-1067. 